\title{
EDITORIAL
}

\section{LA DEPENDENCIA DE LAS PERSONAS CON DISCAPACIDAD: ENTRE LO SANITARIO Y LO SOCIAL, ENTRE LO PRIVADO Y LO PÚBLICO}

\author{
Dolores Puga \\ Instituto de Economía y Geografía. Consejo Superior de Investigaciones Científicas.
}

«De todas las realidades la vejez es quizá aquella de la que conservamos más tiempo en la vida una noción puramente abstracta»

Marcel Proust

El estudio de la dependencia es de gran interés y de máxima actualidad. Se trata de un fenómeno de creciente visibilidad en la sociedad española, debido al fuerte envejecimiento demográfico que está experimentando, y debido también a que sus implicaciones han rebasado el ámbito familiar, al que tradicionalmente se veía confinada. El impacto potencial de esta eventualidad sobre los programas sociales y sanitarios de atención y los altos costes derivados de una situación de dependencia, tanto para los individuos como para sus familias y para la sociedad en su conjunto, explican la creciente atención prestada al tema. Asimismo, los estudios referidos a la dependencia tienen una gran relevancia en el momento actual, de cara a la redacción y discusión del Proyecto de Ley de Protección de la Dependencia que prepara el Ministerio de Asuntos Sociales.

La mayor parte de los análisis sobre la dependencia tienen como finalidad contribuir al diseño de medidas de política sanitaria, política social y de cuidados de larga duración, que hagan posible un aumento del bienestar de las personas en situación de desventaja. En esta línea el presente número de la Revista Española de Salud Pública incluye un estudio sobre la medición de la tasa de dependencia en la población española que plantea una propuesta sobre los criterios de valoración de la misma ${ }^{1}$. Los resultados muestran que si además de la severidad en la valoración se incluye la codiscapacidad se consigue un indicador más refinado, estimando de forma más precisa las situaciones más graves por acumulación de discapacidades. Son estas situaciones, propias de las edades más avanzadas y que presentan una mayor complejidad, las que tenderían a aumentar, de confirmarse el retraso en la aparición de la dependencia encontrado por Otero y colaboradores para la población mayor de Leganés, publicado recientemente en un trabajo en esta misma revista ${ }^{2}$.

Otro paso en la mejora de la medición y valoración podría ser añadir a la severidad a la codiscapacidad, un nuevo componente: una valoración de la carga o peso diferencial que supone la discapacidad para cada una de las actividades observadas dado que, por ejemplo, no es lo mismo no poder comer que no poder hacer la compra. En este sentido, algunos autores han avanzado en la creación de indicadores más complejos que incluyan 
las distintas facetas o componentes de la discapacidad que genera dependencia. Ejemplo de ello es la propuesta de Viciana y colaboradores para el cálculo de la carga de la discapacidad $^{3}$, o el trabajo del AGGIR, realizado para la preparación de la ley francesa de protección de la dependencia, que se basa en la construcción de patrones de discapaci$\mathrm{dad}^{4}$. De cara al establecimiento de un sistema público de protección de la dependencia, resulta extraordinariamente relevante la discusión y mejora de los instrumentos de medida y valoración de la misma.

Entendemos por discapacidad la dificultad para desempeñar papeles y desarrollar actividades socialmente aceptadas, habituales para las personas de similar edad y condición, es decir, la discapacidad es la dificultad o la imposibilidad para llevar a cabo una función o un papel en un contexto social y en un entorno determinado. La discapacidad es la expresión de una limitación funcional, emocional o cognitiva en un contexto determinado. Es la brecha existente entre las capacidades de la persona (condicionadas en parte por su salud) y las demandas del medio (físico, social, laboral). Se vincula más a la función social que a la función orgánica (a la que se asocian patología y deficiencia). Estas dificultades obligan a la persona que las sufre a utilizar dispositivos o bien, principalmente, a solicitar ayuda de otra persona para poder realizar esas actividades cotidianas. En esto consiste la dependencia ${ }^{5,6}$.

Por ello, la dependencia no es únicamente un concepto médico, ni principalmente un problema de salud, sino sobre todo un problema social. La preeminencia del modelo médico a la hora de afrontar tanto el envejecimiento como la discapacidad desvía la atención de la compleja naturaleza del problema y, por tanto, de las soluciones al mismo. Éstas hay que buscarlas no únicamente en las raíces de los problemas de salud sino, sobre todo, en las circunstancias sociales, económicas y físicas del entorno que habita la persona dependiente.
En la actuación frente a la discapacidad y la dependencia resultante se pueden delimitar tres etapas: la prevención, la intervención y la asistencia o intervención paliativa. La primera persigue evitar y retrasar la aparición de la dependencia. La segunda pretende reconstruir la situación de «normalidad» previa, o disminuir el grado de severidad de la misma, una vez que ésta ya afecta al individuo. La tercera actúa frente a sus consecuencias; se trata de dar respuesta a las necesidades ya generadas por la situación de dependencia.

En el ámbito de la prevención, la erradicación de algunos hábitos y costumbres nocivos (tabaquismo, sedentariedad, etc.), muy influenciados por el entorno social del individuo, puede resultar en importantes ganancias en términos de salud y autonomía en la vejez. A ello habría que añadir los resultados positivos que en términos de prevención tendría la mejora de algunas características socio-económicas que resultan muy determinantes de la autonomía a edades avanzadas, como los recursos culturales, económicos, o la fortaleza de la red social ${ }^{7}$.

En el ámbito de la intervención, a la actuación médica, farmacológica o quirúrgica sobre el organismo, habría que añadir intervenciones sobre un entorno físico y social menos exigente, más adaptado a una población tan envejecida como la española actualmente. Queda mucho por hacer respecto a adaptaciones en vías y transportes públicos y, muy especialmente, en los edificios y viviendas en las que residen las personas mayores. Pero éstas no son las únicas adaptaciones necesarias; también las expectativas sociales deben adaptarse a las situaciones de fragilidad creciente propias de la vejez, es decir, también se requieren adaptaciones en las actividades que se esperan de los mayores. Así, por ejemplo, la mayor parte de las mujeres de las actuales generaciones de mayores han de seguir sosteniendo el cuidado de la casa y de los restantes miembros de la familia, aún con sus fuerzas ya debilitadas. 
En el ámbito de la asistencia, a la medicina paliativa se añaden los servicios sociales de respuesta a las situaciones de dependencia, tratando de sustituir o complementar a la persona mayor afectada en aquellas actividades que ella no puede realizar por sí misma. Es en este tercer ámbito en el que la vertiente social de la actuación frente a la dependencia es más conocida, siendo a pesar de ello muy insuficiente y muy reducida frente a la asistencia médica.

Una excesiva medicalización de la dependencia puede resultar costosa para el sistema sanitario y no resuelve los verdaderos problemas de quien pierde autonomía y no puede valerse por sí mismo. Cuando el modelo médico de atención enfatiza el tratamiento clínico y el manejo de las dificultades como enfermedades, estima que las soluciones están más dentro del individuo que de la sociedad, provocando un cierto desentendimiento de la responsabilidad social sobre la dependencia.

Los dos pilares que actualmente en España sostienen la atención a la dependencia en la vejez son la familia y el recurso a la red sanitaria de atención primaria -a veces inadecuado-. Durante mucho tiempo la dependencia ha sido un problema gestionado desde la silenciosa esfera familiar, constituyendo de esta forma un problema de primera magnitud para los individuos y para sus familias, pero poco visible para la sociedad y los poderes públicos. Ello ha derivado en que la atención a la vejez en situación de fragilidad y dependencia -que debería ser un derecho similar al de la atención de la salud- está dominada por una gran desigualdad, puesto que la atención recibida por las personas mayores depende de las circunstancias y capacidades particulares de sus familias. A ello se han de añadir las graves consecuencias que para los familiares tiene la exclusividad de los cuidados, en términos de tiempo -especialmente femenino-, de costes en relaciones, en actividades abandonadas, en oportunidades perdidas -incluso laborales-, y también sobre su propia salud ${ }^{8}$.

De esta forma, hasta ahora la dependencia no ha sido un problema de la sociedad sino un problema de las familias. Este sistema familiarista de bienestar- característico de las sociedades sureuropeas- ha sustentado el envejecimiento de la población española, complementando e incluso sustituyendo en gran medida a los poderes públicos, pero este modelo no es sostenible. La familia seguirá siendo la unidad de referencia incluso cuando los cuidados provengan del exterior, y será un elemento de extraordinario valor como instrumento vehicular entre la atención y la persona dependiente, pero el objeto de protección debe ser el individuo, que en situación de especial vulnerabilidad tiene derecho a ser protegido por la sociedad en la que vive $-y$ que ha ayudado a construir-, tenga o no una familia, o sean cuales fueren las circunstancias de ésta.

Las personas mayores y sus familias suelen llevar sus preocupaciones a la única red que conocen, la sanitaria, que les resulta cercana y en la que confían. La red primaria de atención sanitaria se ha convertido en el primer recurso - no familiar- con el que cuentan los mayores frente a sus dificultades diarias. A esto ha contribuido la ausencia y/o la escasez de otras alternativas, su limitada capacidad para llegar a la población mayor -que, en buena medida, no las conoce-, las dificultades de los procedimientos y, especialmente, la falta de un verdadero modelo social de atención a la dependencia. Las personas mayores acuden a la red primaria de salud en busca de ayuda ante los problemas que dificultan su vida diaria, aunque las respuestas que necesiten sean tanto sociales como sanitarias, como ocurre con la dependencia.

Ésta debe ser abordada necesariamente desde un modelo que integre la atención sanitaria y la social. La reivindicación sostenida de lo «socio-sanitario», sumada a lo 
complejo y costoso de su implementación, y por lo tanto, a su prácticamente nula puesta en práctica, ha «gastado» el término en cierto modo sin prácticamente haberlo estrenado. Pero no por ello se ha de renunciar al fortalecimiento de unos servicios sociales que complementen a los amplios servicios sanitarios existentes, extraordinariamente valiosos y muy bien valorados por los mayores. Dicho fortalecimiento complementaría y reforzaría al mismo tiempo al sistema sanitario, pues lo descargaría de todos aquellos problemas derivados de cuestiones sociales que lo sobrecargan. A ello se ha de añadir que resultaría muy desafortunado pretender que sea la población mayor en una situación de dependencia la que divida sus necesidades en sociales y sanitarias. Debe ser el sistema de protección a la dependencia el que también ha de entender que la respuesta debe ser socio-sanitaria, integrando las soluciones de ambas naturalezas y usando las riquezas ya existentes -como la red sanitaria primaria-, sin pretender que sea la población mayor la que tenga que peregrinar en busca de soluciones parciales. Se presenta una gran oportunidad para ofrecer una respuesta socio-sanitaria a un problema que es, como pocos otros, socio-sanitario.

En el cuidado a la dependencia, la familia ya se encuentra al máximo de su capacidad de cuidados. Conviene trasladar la responsabilidad de la atención a la dependencia a la esfera pública, y la atención en mayor medida hacia la esfera social, basculando entre lo sanitario y lo social, y encontrando la forma de integrarlo en un sistema que los mayores y sus familias conocen bien, sienten cercano, y confían en el. El planteamiento estatal del problema de la dependencia debería abordar el traslado de la responsabilidad desde la familia a la comunidad, y de la atención desde lo médico a lo social.

\section{BIBLIOGRAFÍA}

1. Alegre Escolano A, Ayuso Gutiérrez, Guillén Estany M, Monteverde Verdenelli M, Pociello García E. Tasa de dependencia de la población española no institucionalizada y criterios de valoración de la severidad. Rev Esp Salud Pública 2005; 79:

2. Otero Á, Zunzunegui MV, Rodríguez-Laso Á, Aguilar MD, Lázaro P. Volumen y tendencia de la dependencia asociada al envejecimiento en la población española. Rev Esp Salud Pública 2004; 78:201-213

3. Viciana F, Hernández JA, Canto VD, Ávila AI. Longevidad y calidad de vida en Andalucía. Sevi1la: Instituto de Estadística de Andalucía; 2003

4. Sancho MT. La protección social a las personas mayores dependientes en Francia. Boletín sobre envejecimiento Perfiles y Tendencias 2004; 10:116.

5. Puga MD, Abellán A. El proceso de discapacidad. Un análisis de la Encuesta de Discapacidades, deficiencias y Estado de Salud. Madrid: Fundación Pfizer; 2004

6. Rodríguez Cabrero G (coord.). La protección social de la dependencia. Madrid: IMSERSO; 1999.

7. Rodríguez Laso A. El efecto de las relaciones sociales sobre la mortalidad en las personas mayores. El estudio «Envejecer en Leganés». Tesis doctoral. Departamento de Medicina Preventiva y Salud Pública. Universidad Autónoma de Madrid; 2004

8. Abellán A, Puga MD. La dependencia entre los mayores. En: Informe España 2001. Una interpretación de su realidad social. Madrid: Fundación Encuentro; 2001. p. 187-246. 